\title{
The Employment Difficulties of Retired Athletes in China and Its Path Analysis
}

\author{
Keqing Sheng \\ Wuhan University of Science and Technology \\ Wuhan, China
}

\author{
Furong Yang \\ Wuhan University of Science and Technology \\ Wuhan, China
}

\begin{abstract}
The employment predicament of retired athletes has been exposed frequently and has gradually become one of topics highly valued by the academia in the recent years. This paper is intended to set out from the employment and living conditions of retired athletes to analyze the difficulties and reasons of the employment placement of them and gives advice from three aspects including the self-optimization of athletes, assistance dominated by the government departments and the support from social cooperation, that are, to improve the cultural quality and ability, perfect the social security system and provide various supports in aspects of employment and entrepreneurship. The author hopes that this paper can provide effective reference for relevant researches on the employment of retired athletes in China so as to promote the vigorous development of sports undertakings in China.
\end{abstract}

\section{Keywords -retired athletes; employment; placement}

\section{INTRODUCTION}

The glorious history of sports circles in China relies on the hard efforts of athletes. Nowadays, China has become one of the sports powerful nations, however, the conflict between training and study still remains unsolved and the retirement of athletes means unemployment. Moreover, the placement policies of the government departments have not been thoroughly carried out which makes their livings even rougher. After the 2016 Olympic Games in Rio, there must be a peak of athlete retirement. It is extremely urgent to solve the survival and development problems of retired athletes.

\section{CURRENT EMPLOYMENT STATUS OF RETIRED ATHLETES IN CHINA}

\section{A. The Employment Rate of Retired Athletes Is Not Satisfactory Especially for Those with Illness and the Disabled}

The competitive sports undertakings in China carry out the whole nation system dominated by the national investment. On the one hand, it cultivates batches of athletes with championship which promotes the rapid development of national sports undertakings and industry. On the other hand, it limits the improvement upon the culture learning ability, social skills and other aspects. Although the implementation of placement policies for retired athletes has achieved remarkable effects, the overall successful placement ratio is just $33 \%$. There may spring up the large scale of athlete retirement after the 2016 Olympic Games in Rio, however, the prior retired athletes have not gotten effective placement which exerts cacoethic effects on the competitive sports and even the social stability and order in China. As the special group, after retirement, the athletes will be faced with more severe employment situation and need special treatment and care. The actions in sports program are with high risks and the fall damage and sprain usually happen. Once server accidents happen which will cause the permanent disability of different degrees, they cannot even maintain the basic living, not to mention the obtaining of an occupation to earn a living.

\section{B. New and Old Problems of Retired Athletes Superpose Together Which Makes the Placement Work Even Harder}

There are plenty of retired athletes in China. It is reported that there are 3000 retired athletes each year in China. The problems left over by history plus the new problems make the relevant departments unprepared. With the coming of the market economic system, the retired athletes are now not guaranteed with job assignment. For the current main employment orientation, part elite athletes has obtained placement while part of them go to colleges and universities for further study, while other athletes can only find other way out on themselves. The current employment support and complementary policies aiming at them are still not comprehensive enough and they are still faced with plenty of difficulties which causes many retired athletes cannot make a living. In addition, the sports resources limitation makes the retired athletes nowhere to be placed. The plenty of unemployed retired athletes have a strong impact on the economical running and order of the sports teams.

\section{The Historic Achievements and Educational Level of Retired Athletes Will Directly Influence Their Employment}

Too low diplomas of athletes will directly influence their employment. Among the national athletes, those with the educational level of senior high school account for $50 \%$, those with the educational level of below junior high school account for $46 \%$ and the general compulsory education of retired athletes cannot reach to the average level and the integrated educational level cannot meet the requirements of talents required by the society. The recruitment requirements in the current society basically all need to possess the corresponding technical diplomas or diplomas above the general associate degree while the athletes put all efforts on training for the championship and have few energy focusing on the cultural 
courses and often cannot pass the examinations which greatly reduce the confidence of athletes for further promotion of diplomas after retirement. The retired athletes obtained awards in the international athletic competitions not only can be focused by the public and paid high attention by the government departments, but also can obtain the popularity from the business media and entertainment. And the accompanying advertising and commercial performance open up brand-new ways while those ordinary athletes with average achievements only can find their own places by themselves.

\section{The Executory Effect of Relevant Departments Is Too Weak and the Policies Are Not Thoroughly Implemented}

The employment difficulties of retired athletes need the coordinative cooperation of multipartite leading units and the effectiveness of policies can be exerted. In recent years, the General Administration of Sports of China has issued the a series of documents related to the employment placement for retired athletes and each province should implement one by one according to the regulations. However, the fact is that the execution process involves many departments such as Sports Bureau, Bureau of Finance, Bureau of Labor and Social Security, Bureau of Education, Personnel Bureau and Disability Identification Station etc and from the understanding of documents, collection of data, acceptance of information and allocation of tasks etc to the detailed development of plans, the execution efficiency is weaker and weaker. The implementation of policies is relatively slow and the implementation conditions of each province and city are not the same which seriously delays the progress of difficulties solving.

\section{EMPLOYMENT DIFFICULTIES OF RETIRED ATHLETES IN CHINA}

\section{A. The Cultural Knowledge Level of Retired Athletes Needs to Be Further Perfected}

Owing to occupation demands of athletes, they are difficult to keep balance between further cultural learning and sports training and create strong learning atmosphere so that most of them cannot realize further cultural learning. Moreover, social colleges and universities implement the preferential admission system for elite athletes. At present, enrollment proportion of retired athletes is too low; admission threshold is too high, which is short of universality. In other words, the way of study is only suitable to a small part of retired athletes while most of retired athletes are treated equally with ordinary students and are admitted to colleges and universities based on scores through examinations. Faced with weak culture foundation reality, retired athletes look so feeble. However, cultural education of sports athletes is not only a long-term investment but also a popular support project in sports undertakings, which reflects the objective demand of social civilization progress. Therefore, culture education of sports athletes must be conducted from the basic knowledge and be strengthened step by step.

\section{B. Work-Related Injury Insurance System for Retired Athletes Needs to Be Further Perfected}

Under the current system, the traditional work-related insurance system for related athletes becomes useless. At present, the sports undertaking in China begins to flourish and develops with leaps and bounds. However, sport activities have high risks and athletes with class 3 or above serve disability in China have added up to $7 \%$. Therefore, perfection of the work-related injury insurance system for athletes has become crucial. After athletes suffer severe disability accident, they not only suffer from psychological pain but also may be faced with prejudice and pressure when they apply for jobs in the future. Moreover, when the disease reoccurs after many years, it may cause psychological problem. Although the General Administration of Sport of China has issued the Implementation Regulations of Opinions in 2006, the workrelated injury insurance work for athletes in all provinces has not been implemented fully and has covered narrowly; workrelated injury appraisal and compensation declaration procedures are extremely complicated; compensation standard is low, which is insufficient to cover medical and rehabilitation care expenses. Meanwhile, athletes have poor awareness of purchase of commercial insurance; the disability mutual fund in China is only targeted for elite athletes, which makes many ordinary athletes exhausted because it not only weakens training passion of athletes but also impedes the healthy development of sports human resources and team.

\section{Job Selection of Retired Athletes Is Short of Powerful Security and the Economic Compensation Standard Is Low}

Since all provinces and cities have published different economic compensation methods and regulations for job selection by self-determination of retired athletes, most of provinces and cities successively choose to carry out the momentary placement mode to resettle retired athletes, but the compensation standard is difficult to coverage basic living, family support, and so on of retired athletes. Some retired athletes do not use the compensatory payment properly, which makes momentary resettlement fail to play the due role, and the financial department has limited funds and urgently needs to obtain more funds through other ways. Retired athletes as well as ex-servicemen belong to the special groups and should deserve preferential treatment different from the others. It is found that the employment rate of retired athletes depending on the organization resettlement in Shanghai just accounts for $51 \%$. Therefore, the rest of retired athletes can only find other ways out by themselves. When retired athletes choose to select job thorough self-determination, conduct individual operation, start up a business or engage in trade, they are faced with numerous difficulties and the road is full of hardships. Therefore, the government departments have the obligation to build bridges for reemployment of retired athletes, which is a long-term and arduous task.

\section{Retired Athletes Have Unreasonable Self-Expectation and Are Short of Systematic Career Planning}

Considering athletes have been in the hard training environment and put "all efforts on training for the 
championship" for a long time, they are short of basic knowledge of employment situations in the society, employment skills and so on, become more blurred with the concept of career choices and also have no social work experience. On the contrary, they overemphasize honors they won for the country and put forward unrealistic expectations so that they fail to find suitable jobs in the end. Owing to sudden changes in life style and environment after retirement, athletes are difficult to accept the gap in living conditions and other aspects for a while and transform their roles. Moreover, comparison between themselves from time and time and serious psychological imbalance are much easier to lead to unhealthy psychology and increase reemployment difficulty accordingly, which mainly contributed to the fact that they do not have systematic career planning, fail to correctly recognize their advantages and disadvantages and directly impact the their role transformation during reemployment.

\section{IMPROVE EMPLOYMENT PATH OF RETIRED ATHLETES IN CHINA}

\section{A. Enhancement and Improvement on the Level of Athletes Themselves}

1) Plan career of athletes and lead their role transformation: In other words, in order to ensure smooth employment of athletes, when athletes join the sports team at the initial stage, relevant departments should scientifically plan employment path suitable to all athletes with the combination of summary and analysis of their employment will, actual conditions, special individuality and other characteristics and cultivate their enthusiasm for fight for their own future so that they can get ready in advance. After retirement, athletes need to go with the transformation of roles and concept, pay attention to transformation on mental and concept levels, realize and accept the gap of life before and after retirement, eliminate mental imbalance, correctly locate themselves through analysis of character, demands, experiences and other characteristics and find right direction. When they enter into a new environment, they should foster the concept to try to adapt to it, timely modify and adjust their mental patterns.. Only in this way can they much more smoothly change their roles.

2) Deepen "combination of sports and education" to make culture learning keep pace with sports training: Retired athletes have concentrated on exercise and overseas training or have participated in contests for a long term. As a result, they cannot develop systematic cultural knowledge learning, which seriously impacts the promotion of their academic quality and further restricts their optional employment path after retirement. Therefore, government departments must corporate with colleges and universities as well as education department to mutually renovate double educational pattern "combination with sports and education", boldly explore and innovate implementation approach of unity of learning and training with Chinese characteristics. In this regard, government departments can refer to American management method "combination with sports and education", implement the reward mechanism and arouse their enthusiasm of training along with learning through providing subsidiaries, scholarship and so on. Furthermore, the reward mechanism can be linked to entry qualifications of athletes so that idea of wholeheartedly training can be directly transformed at the source. Besides, period of schooling of athletes can be properly extended to make up their learning time sacrificed owning to busy training so as to ensure that athletes also have culture competitive advantages after retirement.

3) Implement scientific and orderly retired athlete employment training: Government departments can corporate with local colleges and universities or vocational training institutions and entrust the third party to undertake specific training through signing contracts. Government departments should aim at sustainable development of athletes, adhere to the principle of voluntary selection, on-demand training, government leading and social assistance, classify occupations that will be engaged by all athletes, provide relevant skill instructions and training targeted for different occupations so as to enhance professional technical capability of retired athletes and ensure them to smoothly handle new jobs. Training content is the core of employment training task. Therefore, government departments need to conduct research on demands of labor and talent market in advance, collect vocational objectives of retired athletes, analyze and summarize training methods and rules applicable to all retired athletes so as to ensure practicability of employment training.

\section{B. Corporation among Government Departments, Colleges, Universities and Enterprises}

1) Strive to lower the threshold for retired athletes to attend colleges and universities: If retired athletes choose to return to colleges and universities and continue to receive education after retirement, the employment difficulty of retired athletes can be indirectly reduced. According to relevant national regulations, only a few of elite athletes are qualified for being admitted to colleges and universities without entrance examination while the rest of athletes are more difficult to enter colleges and universities for further education. Therefore, government departments should combine with sports department and education departments to mutually discuss and formulate special admission requirements for retired athletes and reasonably relax the standard for retired students to study at colleges and universities. For example, during enrollment, government departments can formulate the policy to level down scores for retired athletes so that they can be equally admitted into colleges and universities along with ordinary students. Class 10 athletes who have achieved outstanding results during training can be directly admitted to universities. Moreover, athletes with music specialty, arts specialty or other specialties can be directly admitted to colleges and universities in the name of specialty and can be trained to be specialty in certain fields.

2) Create talent information files of retired athletes: Firstly, government departments can entrust talent exchange service center to integrate personal data of retired athletes and create real personal files of retired athletes, mainly including their character, specialty, sports orientation engaged, contests participated and awards obtained, school records, career objectives and other information. Retired athletes can select jobs in accordance with their actual situations and demands. Secondly, government departments should be responsible for 
widely collecting social recruitment information, pay attention to job vacancy on enterprises and public institutions, establish smooth contact with related enterprises and public institutions and implement national network management to talent information markets of all provinces and cities throughout the country so that they can timely acquire recruitment information, feedback recruitment information to retired athlete unemployed and provide effective information orientation for both parties. Meanwhile, talent information centers can regularly hold abundant special job fairs and organize diversified talent exchange fairs to promote retired athletes to begin new jobs as early as possible.

3) Establish retired athlete employment assistance associations: Retired athlete employment assistance associations can organize retired athletes employed, mobilize all powers of sports administration departments and advocate all provinces and cities to establish retired athlete employment assistance associations, members of which mainly include retired athletes in the past 2 decades. Retired athletes employed spread over all parts of the country. When they unit each other, they just like an abundant employment information resource database. Moreover, retired athletes have similar experiences. Therefore, they are much easier to understand mutual feelings, communicate and consult with each other and can effectively break down mental disorders existed in employment transformation. Meanwhile, they can spread employment information to help athletes to find employment approaches. Employment associations serve retired athletes and help them select jobs through self-determination. Through actively paying close attention to job vacancy on talent recruitment market, public enterprise and institutions and other units, employment associates can timely feedback information to association members so that they can facilitate retired athletes to find suitable jobs. Employment associations are not established for making profits. Therefore, operation funds of employment associations can only depend on government support and donation from Non-Profit Organizations (NPO) and charitable organizations in the society.

\section{Improvement Measures on the Level of Government Policies}

1) Optimize work-related injury insurance system for retired athletes:

In China, concrete implementation of work-related injury insurance for retired athletes is short of compulsory impetus measures; insurance coverage and level need to be improved. Related departments need to consider and improve the WorkRelated Injury Insurance Regulations, attach special importance to equality between rights and duties, properly increase compensation standards of work-related injury insurance, scientifically clarify appraisal classes of workrelated injury, strengthen protection conscience of rights and interests of athletes, simplify procedures for participating in work-related injury insurance and declaring compensation and popularize and implement all retired athletes of all provinces and areas as much as possible. They should encourage the development of types of special commercial insurance for athletes, standardize the Commercial Insurance System for Athletes and establish different rate bases in accordance with degrees of risks of sports items of athletes so that athletes can make choices based on danger classes of their sports items. Moreover, they should gradually establish a new type of special insurance for certain partial body position of athletes. For example, they should establish a special insurance targeted for knee and ankle of track and field athletes. The Disability Mutual Foundation founded by General Administration of Sport of China and managed by China Sports Foundation belongs to support for the worked-related injury insurance for elite athletes. China Sports Foundation can try to carry out the insurance to ordinary athletes and encourage athletes to pay the disability mutual fund by themselves so as to benefit all the athletes. Meanwhile, China Sports Foundation needs to conduct publicity and education of disability mutual insurance knowledge and popularize the value and significance of it. All provinces and cities need to establish independent sectors to take charge of related work in the local areas and grant material rewards for units who execute the work much better. Furthermore, they should publish expenses and other situation of the disability mutual insurance fee at the end of each year, stick to transparency and justice and accept oversight of social media and the masses.

2) Innovate entrepreneurship preferential policies of retired athletes: Government policy and environment support are particularly crucial for change of predicament of retired athletes who depend on retired pension to make a living. When issuing the entrepreneurship support policy for retired athletes, government departments can refer to related policies for job selection by self-determination for career change cadre of the armed forces in China and make the policy scientific and rationalized as much as possible. For example, industrial and commercial administrative departments can give priority to go through certificates, licenses and related procedures of entrepreneurial retired athletes who conform to specified conditions of the policy; when formulating regulations, related commission charges arising out of entrepreneurship of retired athlete can be deducted or exempted; tax revenue and other deductions and exemptions can be given for those who start their own business. At the same time, the government departments can negotiate and corporate with one bank or more banks to provide interest free loan or low-interest loan for entrepreneurship programs of retired athletes so as to ensure stable and healthy development of their entrepreneurship programs.

3) Increase feasible employment support regulations to promote development of job selection by self-determination: The current support policy for reemployment of retired athletes cannot relieve a large number of retired athletes unemployed. Government departments and the General Administration of Sport of China need to conduct comprehensive research from multiple aspects, clear the guiding principle, adhere to the human-oriented principle and provide feasible reference path for reemployment of retired athletes. For example, they can give one-off rewards to retired athletes who can make a living within one year after retirement so as to promote activity and enthusiasm of athletes. Proper tax revenue deduction and exemption can be given to enterprises or units that help retired athletes settle down so as to encourage enterprise and public institutions to relax the conditions and recruit more retired 
athletes. When appointing retired athletes as national public servants, government departments should assign them to hold public servants in national public institutions, officers in armed forces and other positions and effectively and rationally utilize competent retired athletes with the reference of honors and rewards they obtained in service and other indexes. Certainly, they can guide retired athletes from multiple respects. For example, they can conduct publicity through specialized agencies and expand popularity and influence of retired athletes on the society, which can pass olive branch for retired athletes from different directions.

\section{CONCLUSION}

As one of powerful forces for sustainable development of sports undertakings in China, athletes win brilliance and glory in international sports circles through persistent efforts. After all, they need to live a normal life after retirement. As one of special groups in the society, they are faced with low education level, serious disability, less insurance, difficult job selection and other problems. Therefore, national sports departments and government departments urgently need to promote the system. Moreover, they need to lay emphasis on the balance between training and study, formulate innovative job selection and entrepreneurship guidance policy, improve work-related injury insurance system and placement system and establish a scientific and orderly employment support policy. Only in this way will the situation of retired athletes can be effectively changed and stable and healthy development of the athlete group be promoted.

\section{REFERENCES}

[1] Official Website of General Administration of Sports of China. Temperorary Rules on Disability Mutual Insurance for Elite Athletes. No. [S]2004.525.

[2] Cao Yu. Problems and Countermeasures of Social Security and Reemployment of Atheletes [J]. Sports Culture Guide. December 12, 2011.

[3] Yu Yiqun \& Ji Liu. Resrarch and Analysis of the Placement Status of Retired Athletes in Shanghai [J]. July 2012.

[4] Zhang Guohai. Placement Status of Retired Athletes in China and Countermeasure Research [J]. May 2009.

[5] Zhang Ruihua. Career Planning and Management Researches on Athletes [J]. March 2007. 